\title{
Fast Spectral Correlation Based on Sparse Representation Self-Learning Dictionary and Its Application in Fault Diagnosis of Rotating Machinery
}

\author{
Hongchao Wang $\mathbb{D}^{1}$ and Wenliao $\mathrm{Du}^{2}$ \\ ${ }^{1}$ Mechanical and Electrical Engineering Institute, Zhengzhou University of Light Industry, 5 Dongfeng Road, \\ Zhengzhou 450002, China \\ ${ }^{2}$ Henan Key Laboratory of Intelligent Manufacturing of Mechanical Equipment, Zhengzhou University of Light Industry, \\ Zhengzhou 450002, China \\ Correspondence should be addressed to Hongchao Wang; hongchao1983@126.com
}

Received 25 May 2020; Revised 23 July 2020; Accepted 31 July 2020; Published 18 August 2020

Academic Editor: Lingzhong Guo

Copyright (c) 2020 Hongchao Wang and Wenliao Du. This is an open access article distributed under the Creative Commons Attribution License, which permits unrestricted use, distribution, and reproduction in any medium, provided the original work is properly cited.

\begin{abstract}
Rolling element bearing and gear are the typical supporting or rotating parts in mechanical equipment, and it has important economy and security to realize their quick and accurate fault detection. As one kind of powerful cyclostationarity signal analyzing method, spectral correlation (SC) could identify the impulsive characteristic component buried in the vibration signals of rotating machinery effectively. However, the fault feature such as impulsive characteristic component is often interfered by other background noise, and the situation is serious especially in early weak fault stage. Besides, the traditional SC method has a drawback of low computation efficiency which hinders its wide application to some extent. To address the above problems, an impulsive feature-enhanced method which combines fast spectral correlation (FSC) with sparse representation self-learning dictionary is proposed in the paper. Firstly, the sparse representation self-learning dictionary method-K-means singular value decomposition (KSVD) is improved and the improved KSVD (IKSVD) method is used to denoise the original signal, and the periodic impulses are highlighted. Then, the FSC algorithm is applied on the denoised signal and spectral correlation image could be obtained. Finally, the calculated enhanced envelope spectrum (EES) of the denoised signal is obtained by using the spectral correlation image to identify the accurate fault position. The feasibility and superiority of the proposed method is verified through simulation, experiment, and engineering application.
\end{abstract}

\section{Introduction}

Rolling element bearing and gear are the most commonly used supporting and transmission parts in rotating machinery; meanwhile, they are also the fragile parts. It has great significance in machinery condition monitoring to realize their timely fault feature extraction. Vibration analysis has been used widely in fault feature extraction of rotating machinery [1-6] in engineering due to the reasons that vibration signal is easy to collect and it also contains rich fault feature information. However, early weak fault features of rolling bearing or gear are hard to extract using the traditional signal processing method such as envelope demodulation spectral (EDS) [7] and wavelet transform [8] because the early weak fault features are often overwhelmed by strong interference. In recent years, kinds of methods have been arising to enhance the impulsive components buried in the vibration signal of rotating machinery. One kind of method is mainly focusing on constructing an optimal band-pass filter to denoise the original early weak fault signal of rotating machinery. Kurtogram [9] basing on spectral kurtosis [10] is the pioneer of such methods which uses time-frequency analysis and the kurtosis index to select the resonance frequency band with a large number of impulses. However, there exist some inherent defects in kurtogram. To solve the defect of Kurtogram in extracting the 
repetitive impulses, concepts of spectral negentropy and infogram [11] were introduced to detect the repetitive transients for condition monitoring. Inspired by the idea of spectral kurtosis, the spectral $L 2 / L 1$ norm was verified as a new perspective for spectral kurtosis to characterize the nonstationary repetitive impulsive signals [12]. Spectral kurtosis and envelope were combined for detection of the localized bearing faults in induction machines successfully [13]. Spectral kurtosis was improved and an adaptive spectral kurtosis method was proposed for identification of multiple transient faults [14], and performance of the proposed method was validated by using simulation and experimental signals from a bearing with multiple faults and two faulty bearings. To improve performance of spectral kurtosis in dealing with the situation of low signal-to-noise ratio, a novel method naming protrugram for selection of the optimal frequency band was presented [15], and the effectiveness of the proposed method was validated through simulated and real data.

It is well known that the vibration signal of a defective rolling bearing is typical cyclostationary signal, and its second-order statistic exhibits periodicity [16], and the other kind of method is making use of the cyclic statistical properties of defective bearing vibration signals. SC based on second-order statistic is one kind of typical and powerful tool for feature extraction of cyclostationary signals [17-20]. SC has been used widely in fault diagnosis of rotating machinery in recent years. For example, a novel scheme [21] which combined SC and convolutional neural networks (CNNs) was proposed for fault diagnosis of rolling element bearing, and improved classification performance could be obtained. SC was used for vibration analysis in a wind turbine generator successfully, and the effectiveness of SC with corresponding precautionary measures for protecting bearing in generators is verified and suggested [22]. However, as stated in paper [23], since the transformation from one-dimensional time domain to two-dimensional spectrogram involves a large number of discrete Fourier transform operations and correlations, the traditional SC is very complicated and has a very low efficiency, so the engineering application of SC is very limited. Accordingly, a new method calculating SC named FSC [24] was proposed to solve the above shortcomings of traditional SC. Compared with SC, FSC not only has advantage of higher calculation efficiency, but also could capture cyclostationary signal much more accurately by refining frequency resolution arbitrarily. Kurtosis weighting was introduced to FSC to improve the performance of enhanced envelope spectrum (EES) [25]. However, FSC still has the defect of low robustness to strong background noise as being verified in the paper [23]. The sparse representation self-learning dictionary method such as KSVD could capture the characteristic components hidden in the vibration signal without prior knowledge of the analyzed signal such as wavelet transform. Besides, it has virtue of strong noise robustness and has been applied in fault diagnosis of rotating machinery widely [26-28]. Consequently, a rotating machinery fault diagnosis method by combing sparse representation self-learning dictionary with FSC is proposed in the paper. Firstly, traditional KSVD is improved to improve the adaptability and calculation efficiency of KSVD. Then, the original vibration signal is denoised by the improved KSVD. Subsequently, FSC is applied on the denoised signal, and the obtained results are further processed to obtain EES result to realize enhanced extraction periodic impulse of rotating machinery. The method was proposed due to the following advantages: (1) IKSVD is used for denoising due to the reason that it does not need prior knowledge of the analyzed signal such as wavelet transform. (2) Much better and intuitive fault feature extraction result could be obtained by applying FSC on the denoised signal. (3) The proposed method has much higher computation efficiency over SC though the denoising step is added.

The remaining of the paper is arranged as follows. Theories of IKSVD and FSC are introduced briefly in Section 2 and 3, respectively. The flowchart and details of the proposed method by combing IKSVD with FSC are described in Section 4. Effectiveness of the proposed method is verified by simulation in Section 5 and experiment in Section 6. Besides, an engineering application of the proposed method is also presented in Section 7. Conclusion is obtained at last in Section 8.

\section{IKSVD}

Classical sparse representation self-learning dictionary method-KSVD is the generalization of K-means. The convergence ratios of dictionary atom solution procedures and sparse coding are improved by using optimal matching pursuit (OMP) and singular value decomposition (SVD) together in KSVD, and the reason is that the updating of sparse coefficients and dictionary atom could be synchronized by OMP and SVD.

Over complete dictionary, $D$ is found to make the coefficient $S$ as sparse as possible in KSVD, so that the object signal $Y$ could be represented as sparse as possible:

$$
Y=D S \text {, }
$$

where the trained signal is represented by $Y$ and the over complete matrix is token as $D$. $Y$ is corresponding to the column vectors $\left(s_{1}, s_{2}, \ldots, s_{i}, \ldots, s_{N}\right)$ in $S$, and it is also composed linearly of the columns inDaccording to $s_{i}$. The relationship between $Y$ and $D S$ could be transformed into the mathematical model as shown in the following formula:

$$
\begin{array}{ll}
\min _{D, X} & \left\{\left\|s_{i}\right\|_{l_{0}}\right\}, \\
\text { s.t } \quad & \|Y-D S\|_{l_{2}}^{2} \leq \varepsilon .
\end{array}
$$

Formula (2) could be further replaced by the following formula:

$$
\begin{array}{ll}
\min _{D, X} & \|Y-D S\|_{l_{2}}^{2}, \\
\text { s.t } & \forall i,\left\|s_{i}\right\|_{l_{0}} \leq T_{0} .
\end{array}
$$

In sparse coding stage of the traditional KSVD algorithm, OMP is used to solve the sparse coefficients approximately and formula (2) is set as solution object function 
because of the unknown sparsity of sparse coefficients. So, accuracy of the reconstructed signal is determined by the set residual error $\varepsilon$ to some extent. However, there are not relevant literature studies relating to precision selection of residual error $\varepsilon$ in the KSVD algorithm up to now, which is selected basing on experience usually. Besides, nonconvergence of $\varepsilon$ might be caused by the increase of iteration, which brings out poor reconstruction result of the reconstructed signal. The IKSVD method is proposed in the paper to solve the above problems.

IKSVD uses self-adaptive matching pursuit (SAMP) [28] for sparse coding instead of OMP in KSVD. Sparsity of the sparse coefficients could be described adaptively by changing step length, and the reason is that sparsity of the sparse coefficients does not need to be known. Besides, SAMP has advantage of owing much stricter iteration termination conditions than OMP: residual error $\varepsilon$ and the set iteration number are the two fundamental factors deciding the iteration termination of OMP, and the updated residual error $\varepsilon$ or the updated residual error $\varepsilon$ bigger than the residual error $\varepsilon$ and the set iteration number determine the iteration termination of SAMP. Therefore, much more accurate dictionary atom and sparse coefficients could be obtained by the IKSVD method than the traditional KSVD method.

Besides, the $K$ atoms in dictionary $D^{J}$ do not need to be updated completely, and it is enough to update the $K$ atoms with the greatest influence on the reconstructed signal. The structural similarity [29] of the residual error $\mathcal{E}$ corresponding to $D^{J}$ is introduced and calculated, and equation (4) is the structure similarity calculation equation between signal $x$ and $y$ :

$$
C_{S S}(x, y)=\frac{\left(2 u_{x} u_{y}+c_{1}\right)\left(2 \delta_{x y}+c_{2}\right)}{\left(u_{x}^{2}+u_{y}^{2}+c_{1}\right)\left(\delta_{x}^{2}+\delta_{y}^{2}+c_{2}\right)},
$$

where $u_{x}$ and $u_{y}$ are means of $x$ and $y$, respectively, $\delta_{x}^{2}$ and $\delta_{y}^{2}$ are variances of $x$ and $y$, respectively, $\delta_{x y}$ is covariance of $x$ and $y, c_{1}$ and $c_{2}$ are constants, and $C_{S S}(x, y) \in[0,1]$.

The $C_{S S}$ of residual error $E_{k}$ is the $C_{S S}$ of $Y$ and $\sum_{i \neq k} d_{i} s_{i}^{T}$, and all the $C_{S S}$ corresponding to $E_{1}, E_{2}, \ldots, E$ are compared and calculate, then the residual error $E$ with smallest value of $C_{S S}$ is selected. Both sparse coefficients $s_{k}^{T}$ and corresponding dictionary $d_{k}$ are updated once the minimum residual error $E$ is determined. The blindness of the traditional KSVD algorithm in selecting updated atoms could be avoided by repeating the above stated iteration process.

Main steps of improved KSVD are shown as follows:

Step 1: initialization

Matrix $D^{0} \in \mathrm{R}^{N \times K}$ and iteration value $J=1$ are initialized.

Step 2: sparse coding

Sis solved accurately using the SAMP algorithm as shown in formula (3), and $y_{i}$ could be represented by the obtained sparse coefficients $s_{i}$.

Input: sensing matrix $\Theta$ with size of $M \times N$ and the $M$ dimensional observed signal $y$. The relevant initial values are set as follows: the initial residual error $r_{0}=y$, supporting set $\Omega=\Phi$, step length $L=S=1$, index set $T_{\text {add }}$, the total number of iterations $I$, and the initial iteration number $t=1$.

Main steps of sparse coding are as follows:

(1) The correlation coefficients $u=\left\{u_{j} \mid u_{j}=\right.$ $\left.\left|\left\langle r, \theta_{j}\right\rangle\right|, j=1,2, \ldots, N\right\}$ are calculated, where $\theta_{j}$ is the column vector of $\Theta$, and $L$ maximum values inuare selected and composed as set $T_{\text {add }}=\max _{1 \leq j \leq L}\{|u|\}$.

(2) Update the supporting set $\Omega_{t}=\Omega_{t-1} \cup$ $T_{\text {add }}, \Theta_{\Omega t}=\left\{\theta_{j}\right\}\left(\forall j \in \Omega_{t}\right)$.

(3) The sparse signal $s_{t}=\left(\Theta_{\Omega t}^{T} \Theta_{\Omega t}\right)^{-1} \Theta_{\Omega t}^{T} y$ is calculated and obtained.

(4) The largest absolute value among $s_{t}$ marked as $s_{t L}$ is selected, whose corresponding $\Theta_{\Omega t}$ is marked as $\Theta_{\Omega t L}$, and the corresponding supporting set is marked as $\Omega_{t L}$, that is, $F=\Omega_{t L}$.

(5) Residual error is updated as $r_{\text {tnew }}=y-\Theta_{\Omega t L} s_{t}$.

(6) Stop iteration if there is $r_{\text {tnew }}=0$. Otherwise, update iteration step as $L=L+S$ if there is $\left\|r_{t}\right\|_{2} \geq\left\|r_{t-1}\right\|_{2}$, and continue iteration with going back to step (1). If both of the above two conditions are not satisfied, then $\Omega_{t}=F$ and $r_{t}=r_{t \text { new }}$, and the iteration number is added by 1 .

(7) The nonzero terms in $\Omega_{t}$ are used to reconstruct the sparse signals.

(8) Reconstructed sparse signal $s$ is output.

Step 3: dictionary updating

(1) The used sample set is defined as $w_{k}=\left\{i \mid 1 \leq i \leq N, s_{k}^{T}(i) \neq 0\right\}$

(2) Calculate the residual error $E_{k}$ satisfying $E_{k}=Y-\sum_{i \neq k} d_{i} s_{i}^{T} . C_{S S}$ of $E_{k}$ is also calculated, and the smallest $C_{S S}$ is selected

(3) Only the columns corresponding to $w_{k}$ are selected to restrict $E_{k}$, and the nonzero positions in $E_{k}$ are made be the same as $s_{k}^{T}$, and new $E_{k}$ is obtained which is marked as $E_{k}^{R}$

(4) SVD is used to decompose $E_{k}^{R}=U \Delta V^{T}$, and dictionary atom is updated as $\widetilde{d}_{k}=u_{1}, \widetilde{s}_{k}^{T}=\Delta[1,1] \cdot v_{1}$

(5) Iteration number is updated as $J=J+1$

Step 4: output

Learned dictionary $D^{J}$ is obtained.

\section{FSC}

If the period of autocorrelation function of the nonstationary signal $x(t)$ is time-varying (the period is $T_{0}$ ) which could be represented in equation (5), then the signal could be defined as second-order cyclostationarity:

$$
\begin{aligned}
R_{x}(t, \tau)= & E\left\{x\left(t+\frac{\tau}{2}\right) \cdot x^{*}\left(t-\frac{\tau}{2}\right)\right\}=E\left\{x\left(t+n T_{0}+\frac{\tau}{2}\right)\right. \\
& \left.\cdot x^{*}\left(t+n T_{0}-\frac{\tau}{2}\right)\right\} .
\end{aligned}
$$


$R_{x}(t, \tau)$ in equation (5) could be expressed by Fourier series as shown in the following equation:

$$
R_{x}(t, \tau)=\sum_{\alpha} R_{x}(\tau, \alpha) e^{j 2 \pi \alpha t}
$$

where $\alpha=m / T_{0}, m=\ldots,-2,-1,0,1,2, \ldots$ represents the discrete cyclic frequency and $R_{x}(\tau, \alpha)$ is Fourier coefficients which could be calculated by

$$
R_{x}(\tau, \alpha)=\frac{1}{T_{0}} \int_{-T_{0} / 2}^{T_{0} / 2} R_{x}(t, \tau) e^{-j 2 \pi \alpha t} \mathrm{~d} t,
$$

where $R_{x}(\tau, \alpha)$ is named as cyclic autocorrelation (CA) and $\alpha$ is cyclic frequency $(\mathrm{CF})$. The fault characteristic frequency (FCF) is $\mathrm{CF}$ when fault arises in rolling element bearing.

The Fourier transform of CA relating to time-delay $\tau$ is named SC which could be expressed by

$$
S_{x}(\alpha, f)=\int_{-\infty}^{\infty} R_{x}(\tau, \alpha) e^{-j 2 \pi f \tau} \mathrm{d} \tau .
$$

A one-dimensional signal $x(t)$ is transformed into a twodimensional spectral of $f-\alpha$. The specific cyclostationary components could be extracted from other interferences by applying SC on the vibration signal of faulty rotating machinery. However, the traditional SC has the defects of great computational complexity while dealing with nonstationary vibration signals as stated in the paper [23], which hinders its wide engineering application. To solve the above defect of the above problem of traditional SC, the FSC proposed in the paper [24] is used in the paper, and steps of FSC are stated as follows [24]:

Step 1: parameter initialization

Following parameters are initialized: sampling frequency $F_{s}$; sampling the discrete signal $x[n]$; window $w[n]$ in STFT with the corresponding window length $N_{w}$; and maximum cyclic frequency $\alpha_{\max }$.

Step 2: STFT calculation of the discrete signal $x[n]$

$X_{S T F T}\left(i, f_{k}\right)=\sum_{m=0}^{N_{w}} x[i R+m] w[m] e^{-j 2 \pi m\left(f_{k} / F_{s}\right)}$,

where $f_{k}=k \Delta f=k\left(F_{s} / N_{w}\right)$ and $R=\left(F_{s} / 2 \alpha_{\max }\right)$ represents the block shift in STFT.

Step 3: SC calculation

Initialize $\bar{R}_{w}(\alpha)=0, S\left(\alpha, f_{k}\right)=0, P=\left(N_{w} / 2 R\right), R_{w}$ $(\alpha)=\sum_{n=0}^{N_{w}-1}|w[n]|^{2} e^{-j 2 \pi\left(n-N_{0}\right)\left(\alpha / F_{s}\right)}$, and $N_{0}$ is the central time index of window.

$$
\begin{aligned}
& \text { For } p=0,1,2, \ldots, P, \\
& S\left(\alpha, f_{k} ; p\right)=\sum_{i=0}^{K-1} X_{S T F T}\left(i, f_{k}\right) X_{S T F T}\left(i, f_{k-P}\right)^{*} e^{-j 2 \pi\left(\alpha / F_{s}\right) i R}
\end{aligned}
$$

$$
S\left(\alpha, f_{k}\right)=S\left(\alpha, f_{k}\right)+S\left(\alpha, f_{k} ; p\right) e^{-j 2 \pi N_{0}\left(\alpha / F_{s}\right)-\left(p / N_{w}\right)} .
$$

Step 4: magnitude calibrating

$$
S(\alpha, f)=S\left(\alpha, f_{k}\right) \frac{R_{w}(0)}{\bar{R}_{w}(\alpha) K\|w\|^{2} F_{s}},
$$

where $\|w\|^{2}=\sum_{n=0}^{N_{w}-1}|w[n]|^{2} F_{s}$ and $K$ represents the total number of blocks.

Step 5: SC calculation based on the result obtained in Step 3

Calculate the SC using the following equation:

$$
\gamma(\alpha, f)=\frac{S(\alpha, f)}{\sqrt{S(0, f) S(0, f-\alpha)}} .
$$

Step 6: EES calculation based on the SC obtained in Step 5

$$
S^{E E S}(\alpha)=\int_{f_{1}}^{f_{2}}|\gamma(\alpha, f)| \mathrm{d} f .
$$

\section{Steps of the Proposed Method}

As one of powerful tools to analyze the cyclostationarity signal, SC could extract the periodic impulse features in the vibration signals caused by local faults in rotating machinery such as bearing and gear. However, the periodic impulse characteristic components are often overwhelmed by heavy strong background noise in engineering application or in the early weak fault stage of rotating machinery, and in this situation, the fault features are hard to be extracted. Besides, the calculation efficiency of SC is very low which hinders its wide application in fault diagnosis of rotating machinery. Therefore, FSC based on the sparse representation selflearning dictionary fault diagnosis method is proposed to improve the precision and credibility of rotating machinery fault detection. The steps are described as follows:

Step 1: the IKSVD method is applied on the original vibration signal of rotating machinery, and several selflearned dictionary atoms are obtained

Step 2: reconstruct the vibration signal by using the learned dictionary atoms, and denoised signal is obtained

Step 3: FSC is used to analyze the denoised signal, and the SC image is obtained

Step 4: EES being defined as shown in equation (14) is calculated based on the obtained SC image, and the fault features of rotating machinery are extracted

\section{Simulation}

The feasibility and advantage of the proposed method are verified firstly through the rolling bearing outer race fault simulation signal, and the mathematical model of the simulation signal is shown in equation (15) [30, 31]. In equation (15), the periodic impulse simulation signal of fault 
rolling bearing is represented by $s(t)$, and the added random white noise is token as $n(t)$. T and $\tau_{i}$ represent the period of the impulse signal and tiny fluctuation around $T$, respectively. The fault characteristic frequency of outer race and sampling frequency are set as $f_{o}=25 \mathrm{~Hz}$ and $f_{s}=25.6 \mathrm{kHz} . \tau_{i}$ is the random slide between the rolling element and the race which is assumed to distribute normally, and its value is set as $2 \%$ of the shaft rotating ratio. References $[30,31]$ could be referred to know the meanings of the other parameters:

$$
\left\{\begin{array}{l}
x(t)=s(t)+n(t)=\sum_{i} A_{i} h\left(t-i T-\tau_{i}\right)+n(t), \\
A_{i}=A_{0} \cos \left(2 \pi f_{r} t+\phi_{A}\right)+C_{A} \\
h(t)=e^{-B t} \cos \left(2 \pi f_{n} t+\phi_{\omega}\right) .
\end{array}\right.
$$

Figure 1(a) is time-domain waveform of the simulation signal without adding white noise, from which the periodic impulse character is evident. To simulate the early weak fault of rolling bearing, random white noise with a signal-to-noise ratio of $-10 \mathrm{db}$ is added into the signal as shown in Figure 1(a) and the noised signal is shown in Figure 1(b) from which the impulsive characteristic is buried. EDS is a classical and effective fault diagnosis method for rolling bearing. Apply EDS on the signal as shown in Figure 1(b) directly, and the result is shown in Figure 1(c). The distribution of spectral lines in Figure 1(c) is chaotic, and it is verified that EDS would not work effectively when the fault feature signal of rolling bearing is interfered by strong background noise.

Before applying FSC on the noised signal, the length of the window of Fast-SC is set as $N_{w}=2^{5}$ and the maximum cyclic frequency is set as $\alpha_{\max }=900 \mathrm{Hzs}$ the same as reference [24], and the corresponding FSC analysis results are shown in Figure 2. The outer race fault characteristic frequency $f_{o}=25 \mathrm{~Hz}$ is extracted basing on Figure 2(a) which is the FSC image of the noised signal. The EES analysis result based on Figure 2(a) is shown in Figure 2(b) from which the outer race fault characteristic frequency $f_{o}=25 \mathrm{~Hz}$ is also extracted. However, it is ideal that not only the fault characteristic frequency is extracted, but also its harmonics could be extracted when fault arises in rolling bearings. Furthermore, it is easy to cause misdiagnosis when the fault characteristic frequency of outer race coincides with the rotor rotating frequency.

In the following parts, analysis results of the noised signal as shown in Figure 1(b) using the proposed method are presented. To guarantee the calculation efficiency of IKSVD, the noised signal is divided into several samples and the length of each sample is set as 64 point empirically, and the overlap rate between each sample is set as 0 to avoid the reuse of training signals. Too long length of learned dictionary atoms will affect the calculation efficiency of IKSVD, but too short length could not reflect an impulsive feature completely. So, the length of each learned dictionary atom is set as 256 points in IKSVD considering the above factors. Apply IKSVD on the noised signal as shown in Figure 1(b), and there are a total of 256 dictionary atoms being learned. In the section, parts of the dictionary atoms are presented as being shown in Figure 3. Reconstruct the analyzed signal using the 256 learned dictionary atoms, then apply FSC on the reconstructed signal, and the last analysis results are given in Figure 4. It could be observed that not only the outer race fault characteristic frequency is extracted, but also its harmonics are also extracted being compared with Figure 2 . The feasibility and advantage of the proposed method are verified through simulation.

Since the proposed method IKSVD is mainly developed from the traditional KSVD method, the traditional KSVD and the IKSVD are made as comparison. As analyzed above, the early failure of rolling bearing is manifested as periodic impacts. Therefore, it is the extraction of the occurred impacts which we should focus on. So, kurtosis of the reconstructed signal (KRS) could be used as a good indicator reflecting the denoising effectiveness between the traditional KSVD and IKSVD. Add different levels of white noise into the simulation signal as shown in Figure 1(a), and the signalto-noise ratio (SNR) varies from $-10 \mathrm{db}$ to $2 \mathrm{db}$, and the kurtosis of the reconstructed signal by using traditional KSVD and IKSVD is shown in Figure 5, from which the advantages of the proposed method over the traditional KSVD method are evident. Besides, the calculation efficiency of IKSVD is increased about $25 \%$ compared with KSVD thorough statistics.

\section{Experiment}

In this section, the accelerated bearing life test (ABLT-1A) is carried out and the vibration data of the test bearing' early weak stage is used to further verify effectiveness of the proposed method. Details of the experiment could be referred to reference [32], and they are not stated here due to the space limit. The amplitude and kurtosis of one of the test bearings over its whole life are shown in Figures 6(a) and $6(\mathrm{~b})$. The same as reference [32], the vibration data at $2297^{\text {th }}$ minute are analyzed in the paper to verify effectiveness of the proposed method. Time-domain waveform of the signal at $2297^{\text {th }}$ minute is shown in Figure 6(c) from which the impulse characteristic components could not be identified, and its corresponding EDS analysis result as shown in Figure 6(d) also could not identify the fault characteristic frequency of the test bearing. Besides, the time-domain waveform of the vibration data at $2304^{\text {th }}$ minute with its corresponding EDS analysis result is presented in Figures 6(e) and 6(f), respectively, and the inner race fault characteristic frequency of the test bearing $246 \mathrm{~Hz}$ in Figure 6(f) as stated in reference [31] is not evident. In reality, it is verified through data analysis that the EDS of all the vibration data before $2305^{\text {th }}$ minute all could not reflect the inner race fault characteristic frequency. The time-domain waveform of the vibration data at $2305^{\text {th }}$ minute with its corresponding EDS analysis result is presented in Figures 6(g) and 6(h), respectively, and the inner race fault characteristic frequency $246 \mathrm{~Hz}$ is reflected 


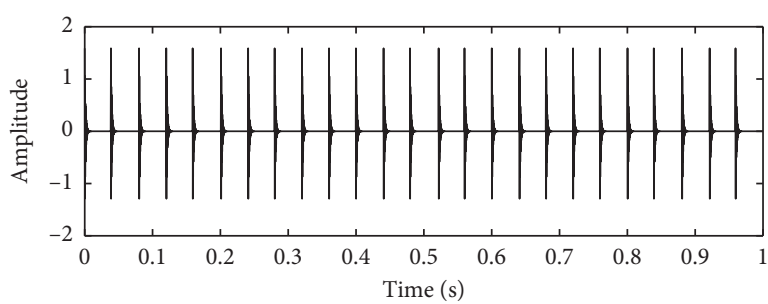

(a)

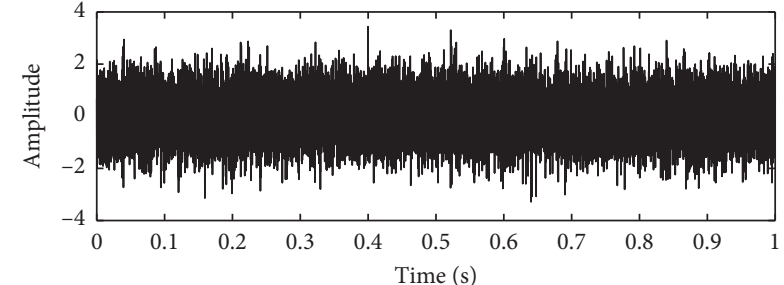

(b)

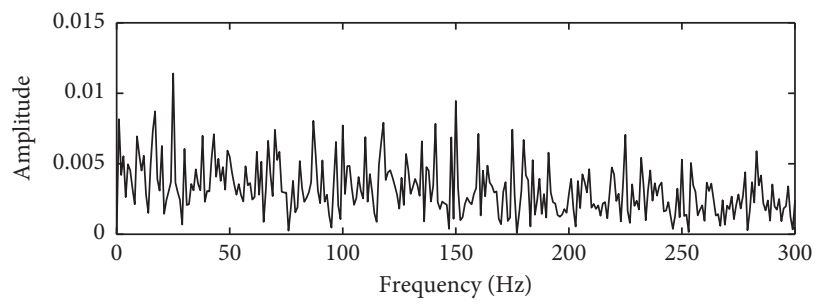

(c)

FIGURE 1: Outer race fault simulation signal of rolling element bearing: (a) simulation signal without noise; (b) simulation signal with strong background noise; (c) EDS of the signal as shown in Figure 1(b).

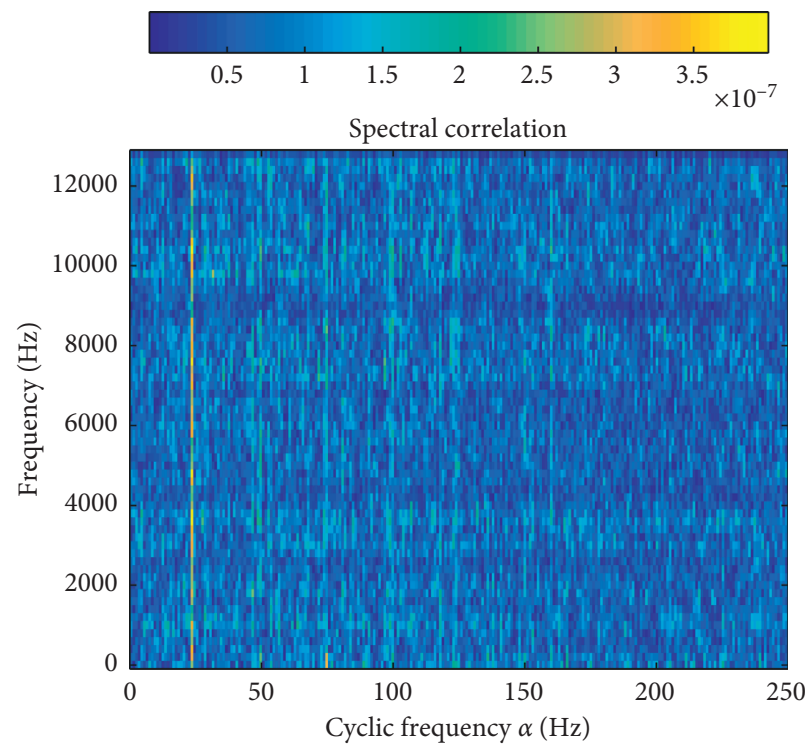

(a)

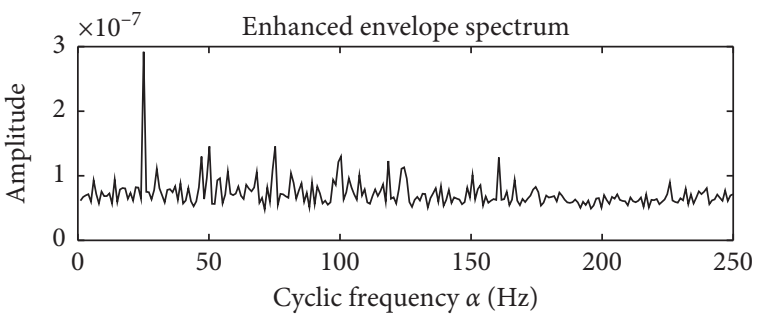

(b)

FIGURE 2: FSC image and EES of the signal as shown in Figure 1(b): (a) FSC image of the signal as shown in Figure 1(b); (b) EES of the signal as shown in Figure 1(b) by using the obtained FSC image as shown in Figure 2(a).

clearly which means the ending of early weak stage of the test bearing at $2305^{\text {th }}$ minute. The vibration data between $2297^{\text {th }}$ minute and $2305^{\text {th }}$ minute could be regarded as early weak fault stage of the test bearing, so it is much more persuasive to use the vibration data at $2297^{\text {th }}$ minute to verify the effectiveness of the proposed method.

The same as the simulation analysis ideology, apply FSC on the signal as shown in Figure 6(c) directly and the corresponding analysis results are presented in Figure 7. However, nothing feature relating to the inner race fault of the test bearing could be observed based on Figures 7(a) and 7(b). Besides, it might lead to misjudgment of outer race fault of test bearing based on Figure 7(b) because the spectral line at $153 \mathrm{~Hz}$ is very evident, and $153 \mathrm{~Hz}$ is exactly the outer race fault characteristic frequency of the test bearing. Actually, it has been proved in reference [31] that failure arised in the inner race of the test bearing through disassemblement of test bearing, and the picture of the actual fault position on the testing bearing is presented in Figure $7(\mathrm{c})$. The reasons for the failure feature extraction of FSC on the 

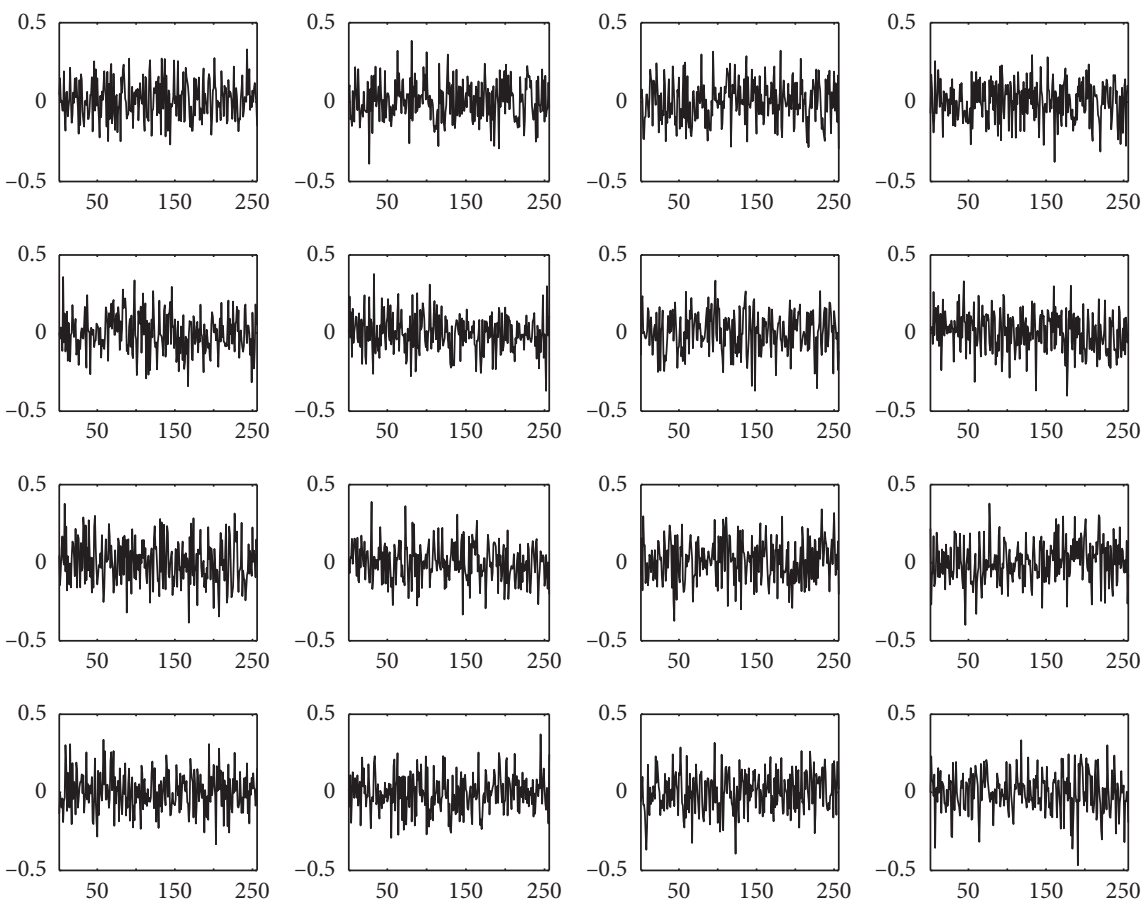

FIgURE 3: Part of the learned dictionary atoms by using the IKSVD method on the signal as shown in Figure 1(b).

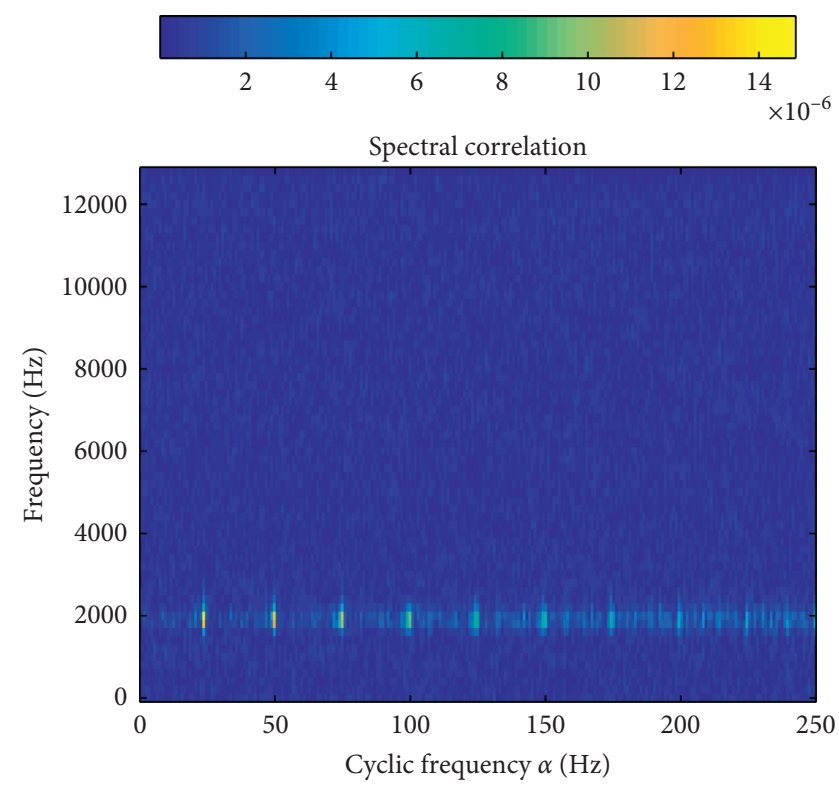

(a)

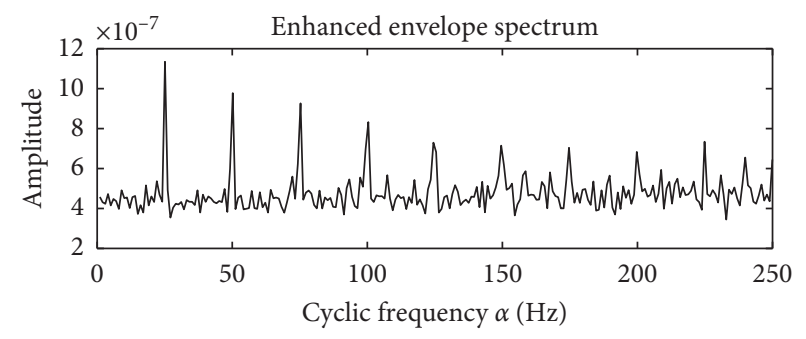

(b)

FIGURE 4: FSC image and EES of the reconstructed signal: (a) FSC image of the reconstructed signal by using the learned atoms as shown in Figure 3; (b) EES image of the reconstructed signal by using the obtained FSC image as shown in Figure 4(a).

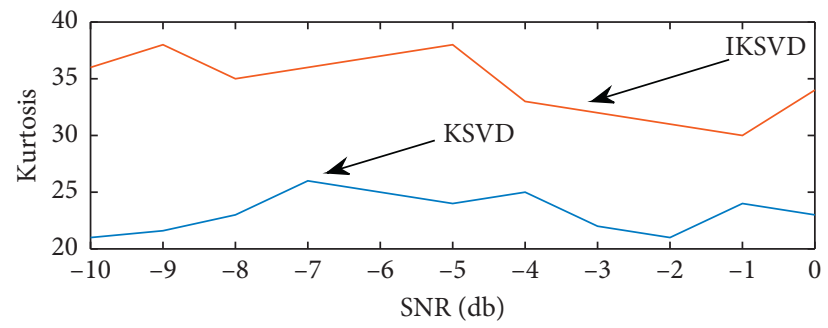

FIGURE 5: The kurtosis of the reconstructed signal by using IKSVD and traditional KSVD. 


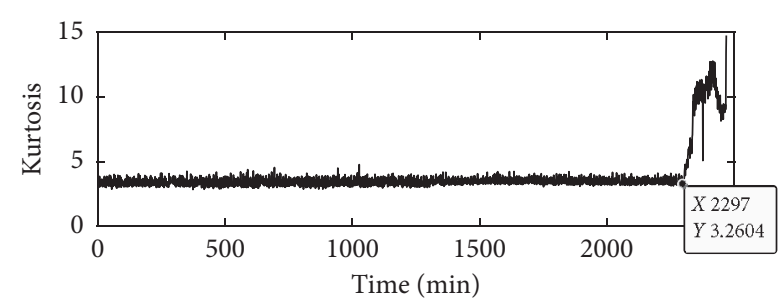

(a)

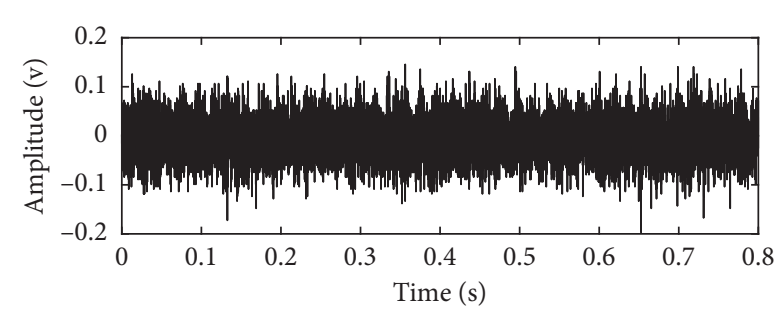

(c)

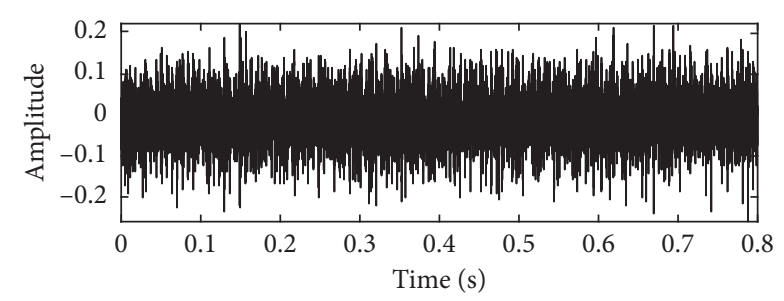

(e)

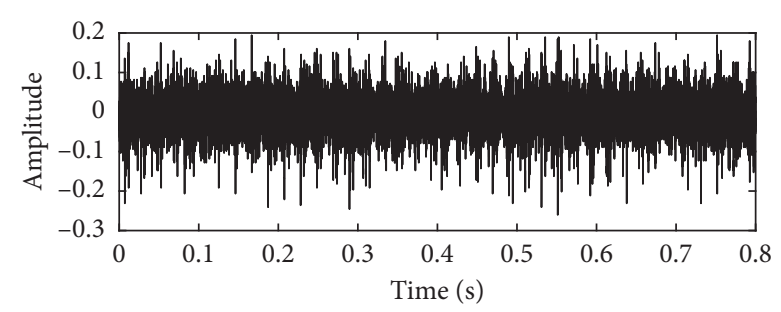

(g)

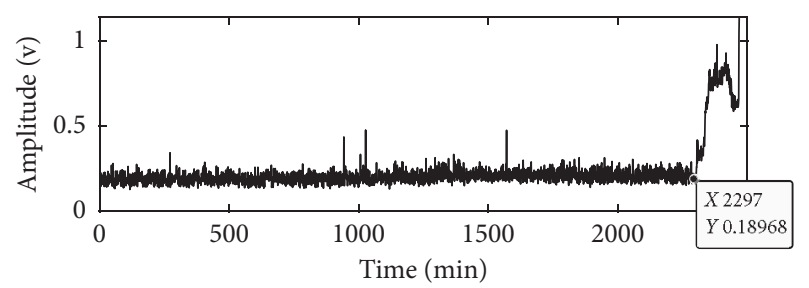

(b)

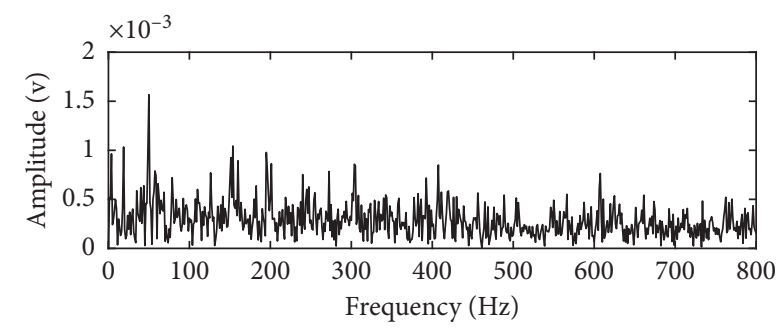

(d)

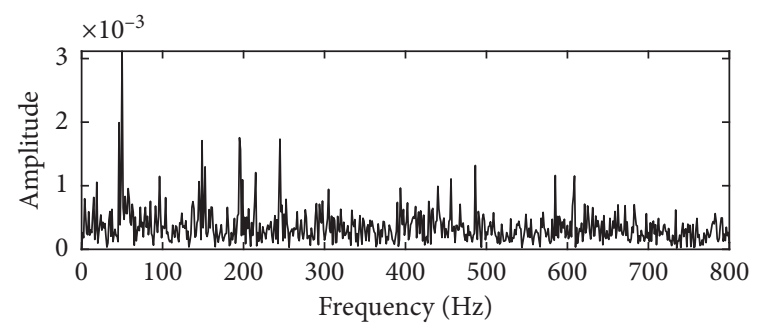

(f)

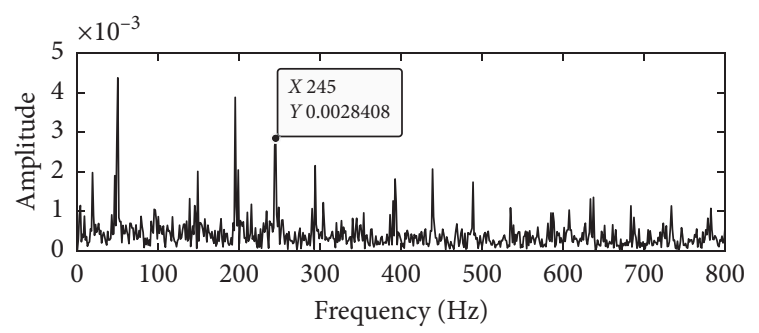

(h)

FiguRE 6: Early weak fault signal of test bearing: (a) kurtosis over its whole life of the test bearing; (b) amplitude over its whole life of the test bearing; (c) time-domain waveform of the signal at $2297^{\text {th }} \mathrm{min}$; (d) EDS of the signal as shown in Figure 6(c); (e) time-domain waveform of the signal at $2304^{\text {th }} \mathrm{min}$; (f) EDS of the signal as shown in Figure 6(e); (g) time-domain waveform of the signal at $2305^{\text {th }}$ min; (h) EDS of the signal as shown in Figure 6(g).

test bearing early weak fault could be attributed to as follows: (1) FSC owns strong ability to supress random white noise as having been verified in reference [24], and it could also be verified in the simulation section. However, the interferences such as the rotating frequency with its harmonics could not be regarded as random white noise relating to the rolling bearing impulsive characteristic components. (2) The energy of impulsive characteristic components of testing bearing at early stage is very weak compared with the other interferences.

Apply the proposed method on the signal as shown in Figure 6(c). Firstly, parameters of IKSVD are set the same as simulation. Then, apply IKSVD on the signal as shown in Figure 6(c) and parts of the 256 learned dictionary atoms are shown in Figure 8. Reconstruct the signal using the 256 learned dictionary atoms, and the reconstructed signal is analyzed by using FSC at last. The final analysis results are given in Figure 9. Firstly, though the FSC could not extract the inner race fault feature directly, the time-frequency analysis is improved greatly by comparing Figure 9(a) with Figure 7(a). Besides, the inner race fault characteristic frequency with its harmonics is extracted perfectly basing on Figure 9(b), and the effectiveness of the proposed method in fault feature extraction of rolling bearing is further verified. 


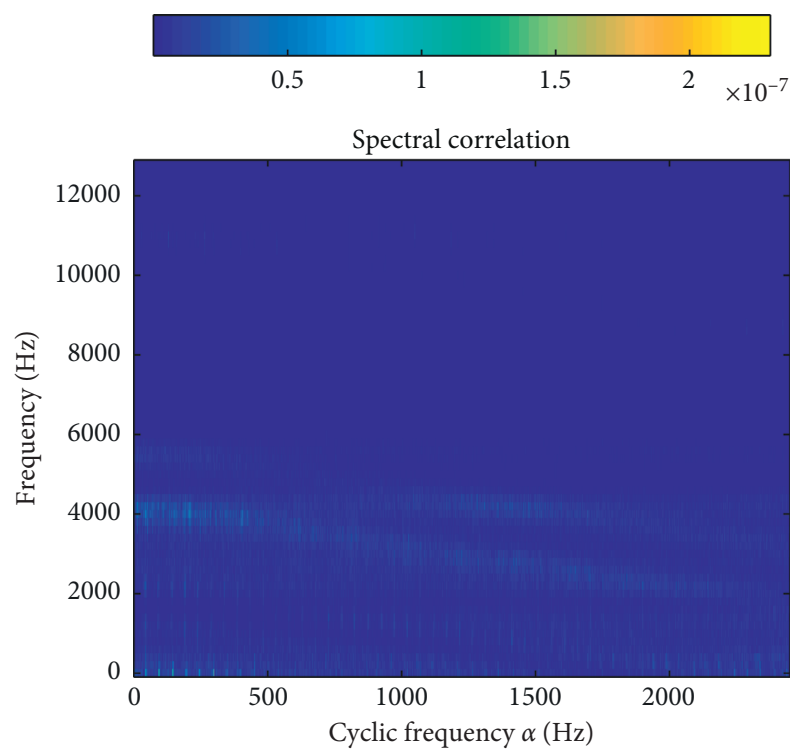

(a)

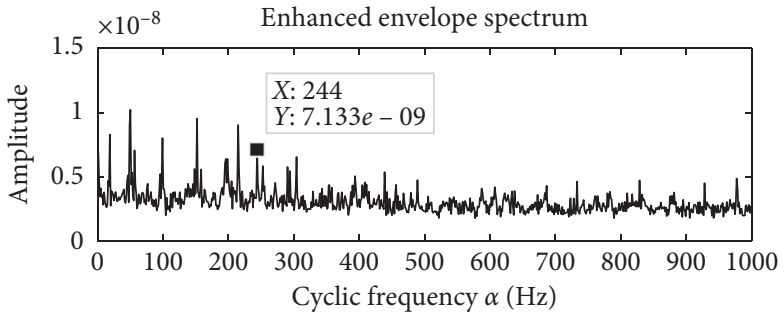

(b)

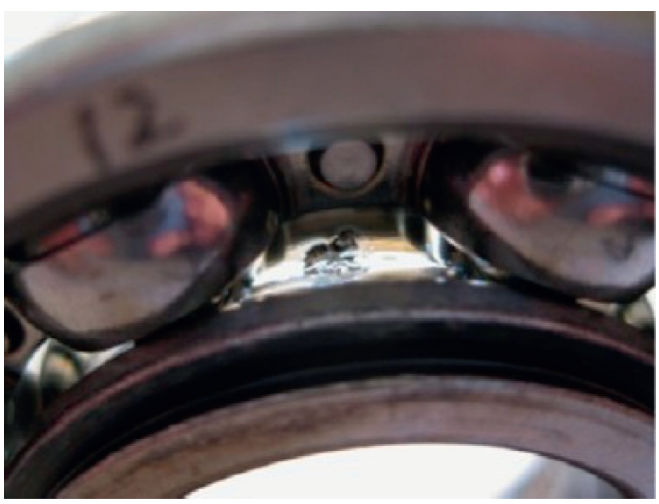

(c)

FIGURE 7: FSC image and EES of the signal as shown in Figure 6(c): (a) FSC image of the signal as shown in Figure 6(c); (b) EES of the signal as shown in Figure 6(c) by using the obtained FSC image as shown in Figure 7(a); (c) actual fault position of the testing bearing.

\section{Engineering Verification}

In this section, the engineering application of the proposed method is presented and the engineering study object is a coal mill in a cement plant, and the structure of the coal mill is shown in Figure 10. The speed of the motor is decelerated by a three-stage gearbox, and then the output speed of the gearbox drives the small gear as noted in Figure 10 through a coupling. The output speed of the motor is $744 \mathrm{r} / \mathrm{min}$, and the transmission ratio of the gearbox is $i=5.6$, so the output speed of the gearbox is $132 \mathrm{r} / \mathrm{min}$. The parameters of the open gears supported by the bearing pedestal are shown in Table 1. It was found that the free end and drive end of the rolling bearing pedestal had a large vibration phenomenon. The measuring points with different directions and the corresponding measuring values are shown in Table 2.
The sensor is the vibration accelerator. The driving end of the bearing pedestal in the horizontal direction with biggest vibration value $14.39 \mathrm{~m} / \mathrm{s}^{2}$ is selected for analysis. Since both the rotating frequencies and the meshing frequency are low, the sampling could be set slightly low, that is, $f_{s}=853 \mathrm{~Hz}$. The length of the sampling data is set as $L=32768$ points to obtain high frequency resolution. Time-domain waveform of the analyzed signal is shown in Figure 11(a) from which the slight modulation and impulse phenomenon could be observed, and its EDS result is shown in Figure 11(b). However, the fault characteristic frequency could not be observed. Then, apply FSC on the signal as shown in Figure 11(a) directly and the corresponding results are presented in Figure 12. Similarly, the fault feature extraction result is not ideal due to the interference of background noise. Apply the proposed method on the analyzed. Firstly, handle the original analyzed signal using the IKSVD method and parts 

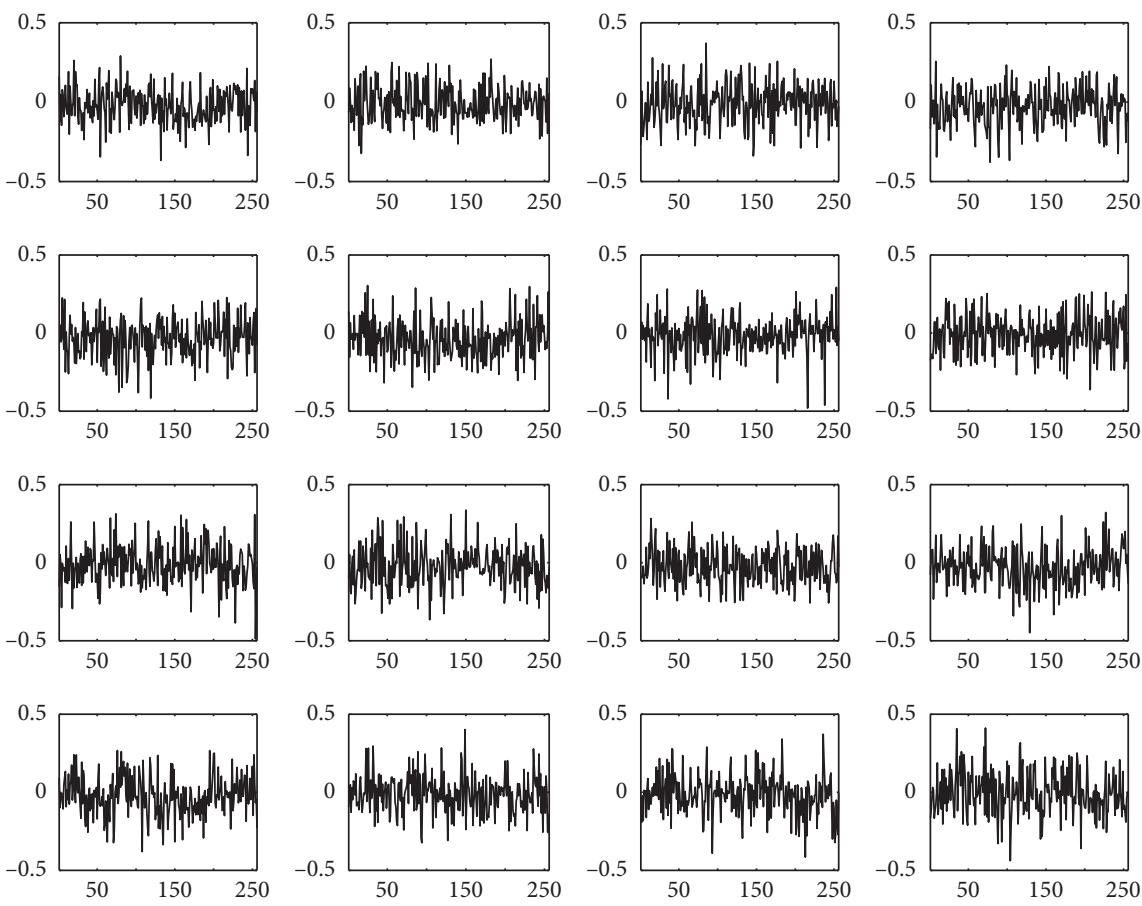

Figure 8: Part of the learned dictionary atoms by using the IKSVD method on the signal as shown in Figure 6(c).

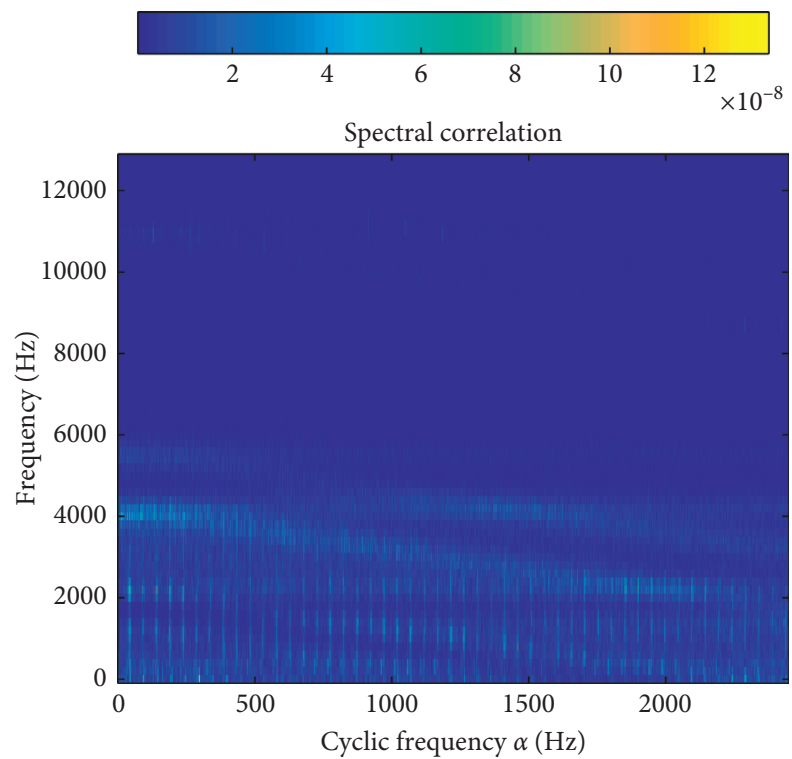

(a)

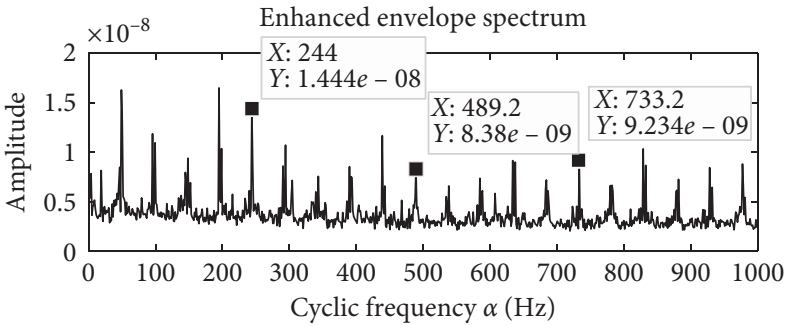

(b)

FIGURE 9: FSC image and EES of the reconstructed early weak fault signal: (a) FSC image of the reconstructed early weak fault signal by using the learned atoms as shown in Figure 8; (b) EES of the reconstructed early weak fault signal by using the obtained FSC image as shown in Figure 9(a). 


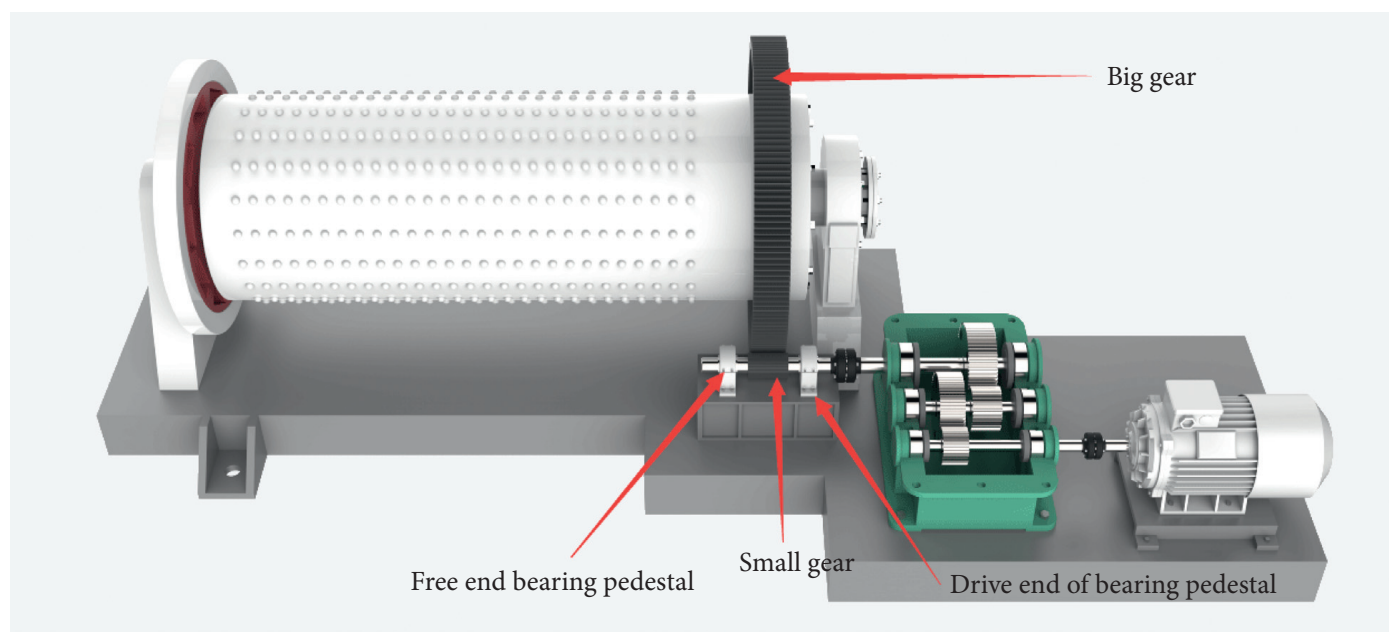

Figure 10: Structure of coal mill.

TABle 1: Parameters of the open gears.

\begin{tabular}{lccc}
\hline Gear name & Tooth number & Rotating speed $(\mathrm{Hz})$ & Mesh frequency $(\mathrm{Hz})$ \\
\hline Big gear & 241 & 0.247 & 59.4 \\
Small gear & 27 & 2.2 & \\
\hline
\end{tabular}

TABLE 2: Values of the measuring points.

\begin{tabular}{lccc}
\hline Number & Measuring points & Direction & Measuring values $\left(\mathrm{m} / \mathrm{s}^{2}\right)$ \\
\hline 1 & & Horizontal & 14.37 \\
2 & Drive end of bearing pedestal & Vertical & 12.29 \\
3 & & Axial & 8.91 \\
\hline 4 & & Horizontal & 10.72 \\
5 & Free end of bearing pedestal & Vertical & 9.1 \\
6 & & Axial & 6.29 \\
\hline
\end{tabular}

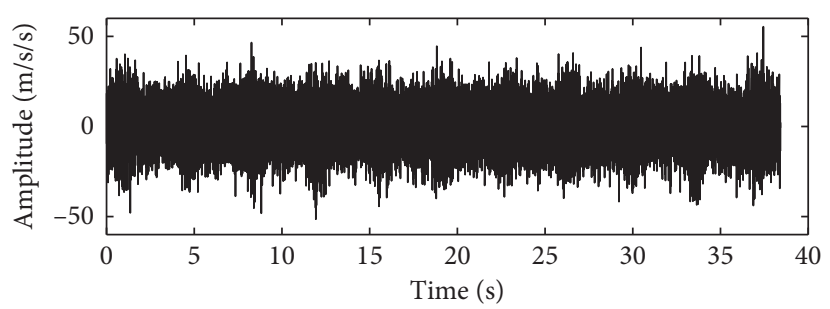

(a)

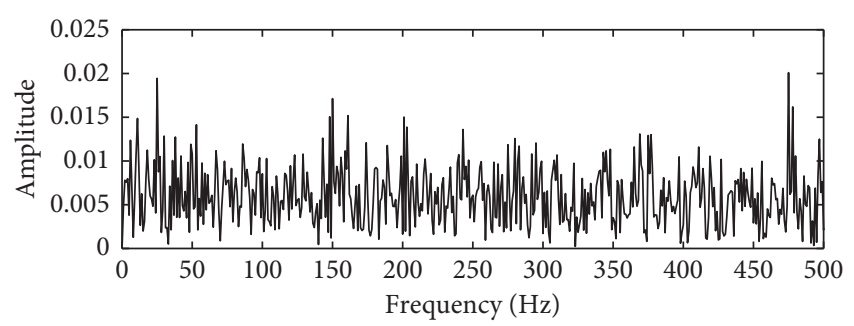

(b)

Figure 11: Vibration signal of the drive end of bearing pedestal measuring point in horizontal direction: (a) time-domain waveform of the vibration signal; (b) EDS of the vibration signal as shown in Figure 11(a).

of the learned dictionary atoms are given in Figure 13, and parameters of IKSVD are set the same as experiment. Then, reconstruct the analyzed signal using the learned dictionary atoms. Subsequently, apply FSC on the reconstructed signal, and the obtained FSC analysis results are given in Figure 14. The meshing frequency and its harmonic components of the open gears are extracted effectively according to Figure 14(a), and the effectiveness 


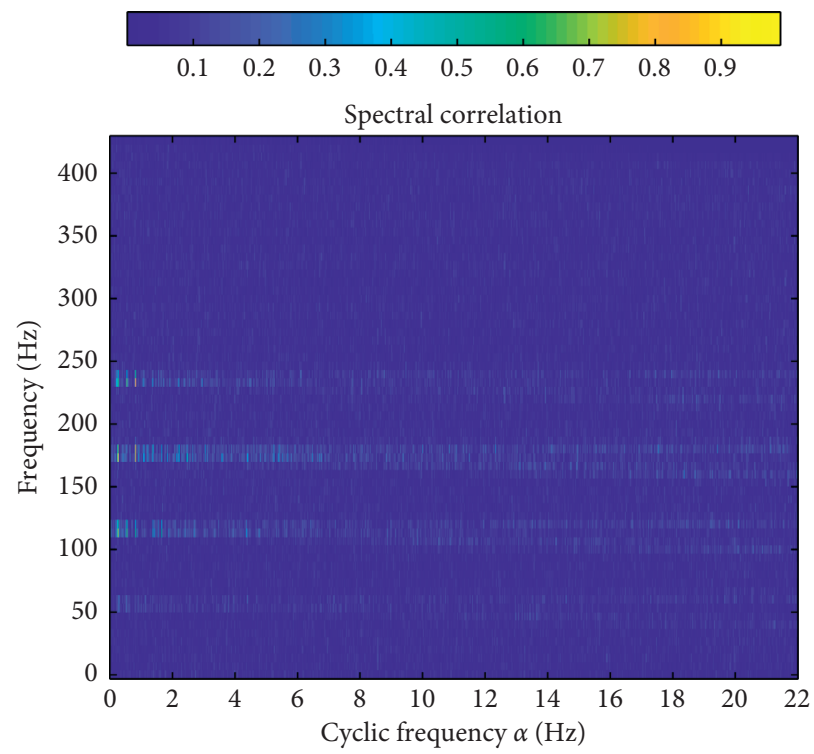

(a)

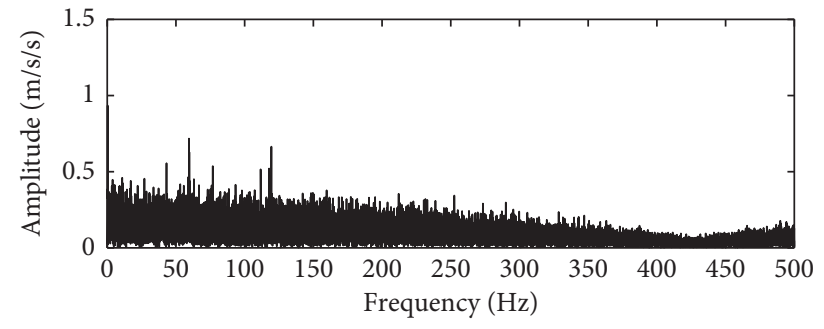

(b)

FIGURE 12: FSC image and EES of the signal as shown in Figure 12(a): (a) FSC image of the signal as shown in Figure 11(a); (b) EES of the signal as shown in Figure 12(a) by using the obtained FSC image as shown in Figure 12(a).
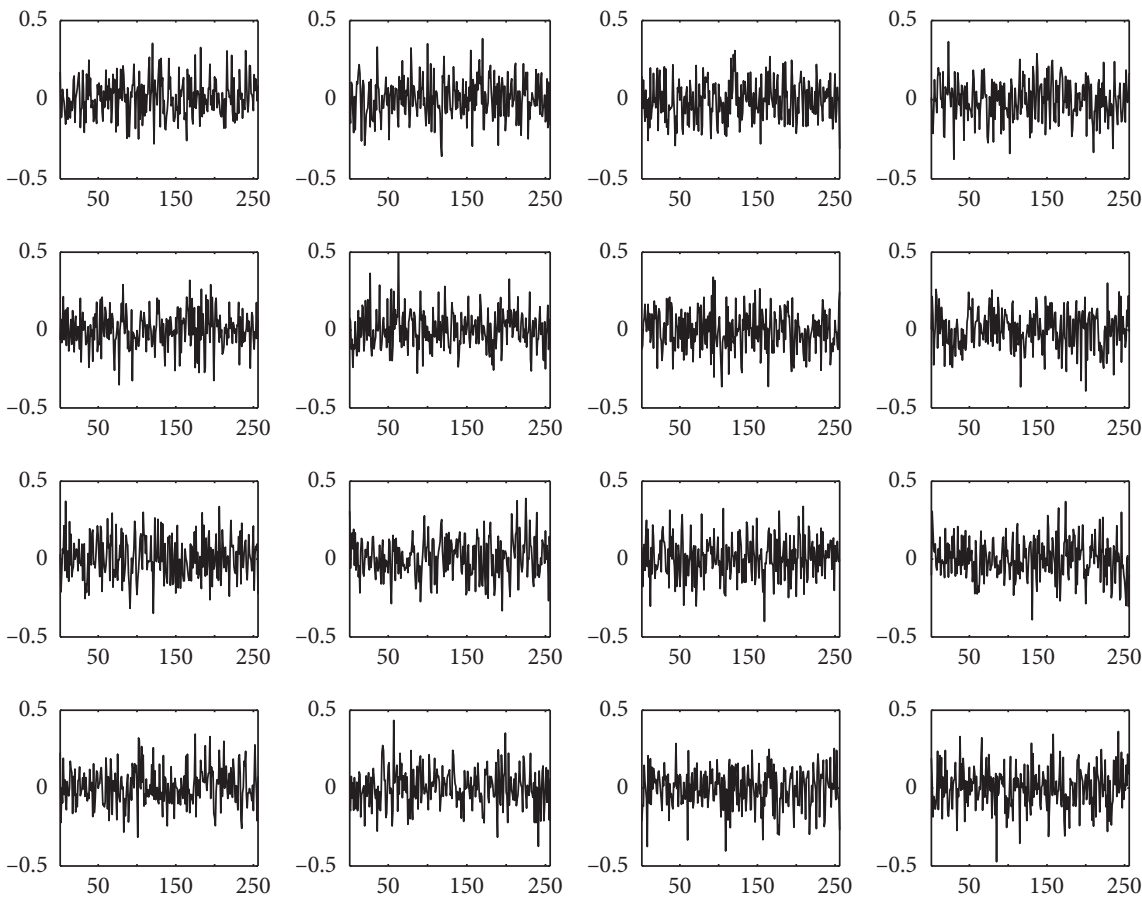

Figure 13: Part of the learned dictionary atoms by using the IKSVD method on the signal as shown in Figure 12(a).

of the proposed method in engineering application is verified. Besides, the horizontal coordinate interval of each spectral line is about $2.2 \mathrm{~Hz}$ which is exactly the rotation frequency of the small gear. The EES of the reconstructed gear signal as shown in Figure 14(b) by using the obtained FSC image as shown in Figure 14(a) is much more intuitive. It could be judged that fault arises in the small gear. Through the disassemblement of the mesh gears, it is found that there exists wear fault on the small gear tooth, which is shown in Figure 15. 


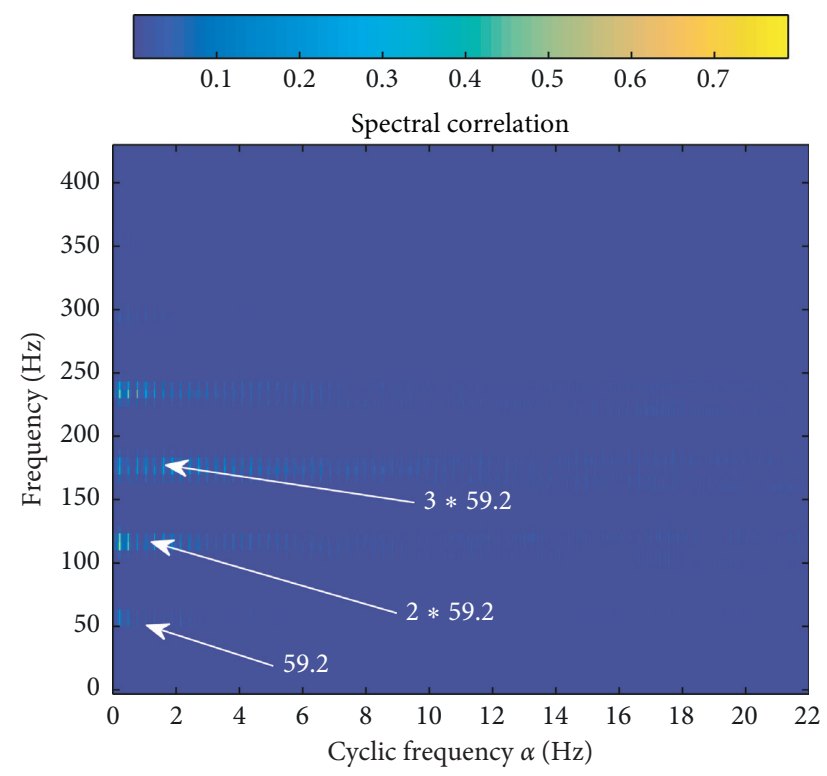

(a)

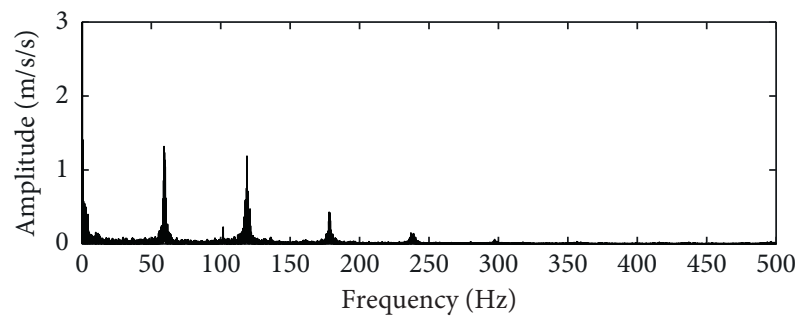

(b)

FIGURE 14: FSC image and EES of the reconstructed gear signal: (a) FSC image of the reconstructed gear signal by using the learned atoms as shown in Figure 13; (b) EES of the reconstructed gear signal by using the obtained FSC image as shown in Figure 14(a).

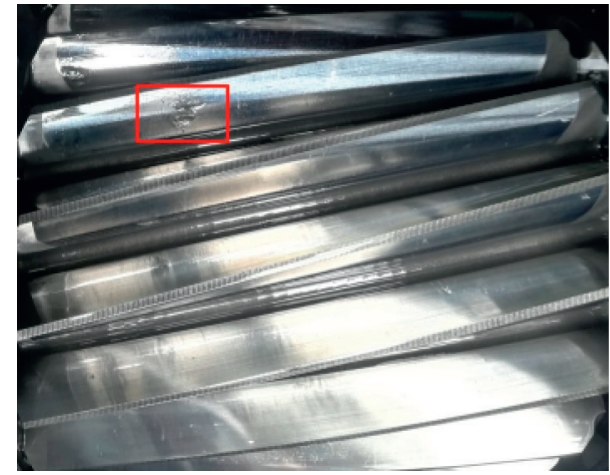

FIGURE 15: Fault location occurring on the small gear.

\section{Conclusion}

In conclusion, a FSC based on the sparse representation selflearning dictionary method was proposed in the paper which is used in fault diagnosis of rotating machinery, and quick and reliable fault detection could be realized. Firstly, the traditional KSVD method was improved, and the IKSVD was used to enhance the impulse signals of rotating machinery. Compared with the traditional KSVD method, IKSVD has the advantage of much more adaptability and higher calculation efficiency by introducing the self-adaptive matching pursuit (SAMP) instead of OMP in the IKSVD method. Secondly, the original signal is reconstructed by the learned dictionary atoms and the impulse feature components buried in the strong background noise are enhanced. Thirdly, the FSC is applied on the reconstructed signal to further highlight the cyclic frequency features of rotating fault frequency and its multiples. Finally, some simulation data, experimental data, and engineering data were analyzed by using the proposed method and other methods such as EDS and FSC directly. The analysis results verified that the proposed method had the advantage in the periodic impulse extraction of rotating machinery over the other methods and could be available for the fault detection of rotating machinery in engineering practice.

\section{Data Availability}

The echelon requires that the current data be kept secret.

\section{Conflicts of Interest}

The authors declare that there are no conflicts of interest.

\section{Acknowledgments}

The research was supported by the National Natural Science Foundation (approved grant no. U1804141) and the Key Science and Technology Research Project of the Henan Province (approved grant no. 192102210105).

\section{References}

[1] L. Mubaraali, N. Kuppuswamy, and R. Muthukumar, "Intelligent fault in microprocessor systems for vibration analysis in roller bearings in whirlpool turbine generators real time processor applications," Microprocessor and Microsystems, vol. 76, Article ID 103097, 2020.

[2] G. Purushottam and T. Rajiv, "Signal based condition monitoring techniques for fault detection and diagnosis of induction motors: a state-of-the-art review," Mechanical Systems and Signal Processing, vol. 144, Article ID 106908, 2020. 
[3] Z. Meng, X. Zhan, J. Li, and Z. Pan, "An enhancement denoising autoencoder for rolling bearing fault diagnosis," Measurement, vol. 130, pp. 448-454, 2018.

[4] T. Han, C. Liu, L. Wu, S. Sarkar, and D. Jiang, "An adaptive spatiotemporal feature learning approach for fault diagnosis in complex systems," Mechanical Systems and Signal Processing, vol. 117, pp. 170-187, 2019.

[5] T. Han, D. Jiang, Y. Sun, N. Wang, and Y. Yang, "Intelligent fault diagnosis method for rotating machinery via dictionary learning and sparse representation-based classification," Measurement, vol. 118, pp. 181-193, 2018.

[6] Y. Hao, L. Song, B. Ren, H. Wang, and L. Cui, "Step-by-step compound faults diagnosis method for equipment based on majorization-minimization and constraint SCA," IEEE/ ASME Transactions on Mechatronics, vol. 24, no. 6, pp. 2477-2487, 2019.

[7] Y. Ming, J. Chen, and G. Dong, "Weak fault feature extraction of rolling bearing based on cyclic wiener filter and envelope spectrum," Mechanical Systems and Signal Processing, vol. 25, no. 5, pp. 1773-1785, 2011.

[8] J.-D. Wu and J.-J. Chan, "Faulted gear identification of a rotating machinery based on wavelet transform and artificial neural network," Expert Systems with Applications, vol. 36, no. 5, pp. 8862-8875, 2009.

[9] J. Antoni and R. B. Randall, "The spectral kurtosis: application to the vibratory surveillance and diagnostics of rotating machines," Mechanical Systems and Signal Processing, vol. 20, no. 2, pp. 308-331, 2006.

[10] J. Antoni, "Fast computation of the kurtogram for the detection of transient faults," Mechanical Systems and Signal Processing, vol. 21, no. 1, pp. 108-124, 2007.

[11] J. Antoni, "The infogram: entropic evidence of the signature of repetitive transients," Mechanical Systems and Signal Processing, vol. 74, pp. 73-94, 2016.

[12] D. Wang, "Spectral L2/L1 norm: a new perspective for spectral kurtosis for characterizing non-stationary signals," Mechanical Systems and Signal Processing, vol. 104, pp. 290-293, 2018.

[13] V. C. M. N. Leite, J. G. Borges da Silva, G. F. C. Veloso et al., "Detection of localized bearing faults in induction machines by spectral kurtosis and envelope analysis of stator current," IEEE Transactions on Industrial Electronics, vol. 62, no. 3, pp. 1855-1865, 2015.

[14] Y. Wang and M. Liang, "Identification of multiple transient faults based on the adaptive spectral kurtosis method," Journal of Sound and Vibration, vol. 331, no. 2, pp. 470-486, 2012.

[15] T. Barszcz and A. JabŁoński, "A novel method for the optimal band selection for vibration signal demodulation and comparison with the kurtogram," Mechanical Systems and Signal Processing, vol. 25, no. 1, pp. 431-451, 2011.

[16] I. Antoniadis and G. Glossiotis, "Cyclostationary analysis of rolling-element bearing vibration signals," Journal of Sound and Vibration, vol. 248, no. 5, pp. 829-845, 2001.

[17] J. Antoni, "Cyclic spectral analysis in practice," Mechanical Systems and Signal Processing, vol. 21, no. 2, pp. 597-630, 2007.

[18] A. Mahvash and A. A. Lakis, "Application of cyclic spectral analysis in diagnosis of bearing faults in complex machinery," Tribology Transactions, vol. 58, no. 6, pp. 1151-1158, 2015.

[19] H. Sun, S. Yuan, and Y. Luo, "Cyclic spectral analysis of vibration signals for centrifugal pump fault characterization," IEEE Sensors Journal, vol. 18, no. 7, pp. 2925-2933, 2018.

[20] J. Antoni, "Cyclic spectral analysis of rolling-element bearing signals: facts and fictions," Journal of Sound and Vibration, vol. 304, pp. 487-529, 2007.
[21] Z. Chen, A. Mauricio, W. Li, and K. Gryllias, “A deep learning method for bearing fault diagnosis based on cyclic spectral coherence and convolutional neural networks," Mechanical Systems and Signal Processing, vol. 140, p. 106683, 2020.

[22] W. Teng, X. Ding, Y. Zhang, Y. Liu, Z. Ma, and A. Kusiak, "Application of cyclic coherence function to bearing fault detection in a wind turbine generator under electromagnetic vibration," Mechanical Systems and Signal Processing, vol. 87, pp. 279-293, 2017.

[23] J. M. Li, Q. W. Yu, X. D. Wang et al., “An enhanced rolling bearing fault detection method combing sparse code shrinkage denoising with fast spectral correlation," ISA Transactions, vol. 102, pp. 335-346, 2020.

[24] J. Antoni, G. Xin, and N. Hamzaoui, "Fast computation of the spectral correlation," Mechanical Systems and Signal Processing, vol. 92, pp. 248-277, 2017.

[25] G. Tang, B. Pang, T. Tian, and C. Zhou, "Fault diagnosis of rolling bearings based on improved fast spectral correlation and optimized random forest," Applied Sciences, vol. 8, no. 10, pp. 1859-22, 2018.

[26] K. Zheng, T. Li, Z. Su, J. Wen, B. Zhang, and Y. Zhang, "Faults diagnosis of rolling bearings based on shift invariant K-singular value decomposition with sensitive atom nonlocal means enhancement," Measurement, vol. 135, pp. 836-851, 2019.

[27] Z. Zhao, B. Qiao, S. Wang, Z. Shen, and X. Chen, “A weighted multi-scale dictionary learning model and its applications on bearing fault diagnosis," Journal of Sound and Vibration, vol. 446, pp. 429-452, 2019.

[28] X. Bi, X. D. Chen, and Y. Zhang, "Variable step size stagewise adaptive matching pursuit algorithm forimage compressed sensing," in Proceedings of IEEE International Conference on Signal Processing,Communication and Computing, pp. 1-4, IEEE, Kunming, Yunnan, China, August 2013.

[29] Z. Wang, A. C. Bovik, H. R. Sheikh et al., "Image quality assessment:from error visibility to structural similary," IEEE Trans. on Image Processing, vol. 13, pp. 600-612, 2014.

[30] P. D. McFadden and J. D. Smith, "Model for the vibration produced by a single point defect in a rolling element bearing," Journal of Sound and Vibration, vol. 96, no. 1, pp. 69-82, 1984.

[31] P. D. McFadden and J. D. Smith, "The vibration produced by multiple point defects in a rolling element bearing," Journal of Sound and Vibration, vol. 98, no. 2, pp. 263-273, 1985.

[32] H. Wang, J. Chen, and G. Dong, "Feature extraction of rolling bearing's early weak fault based on EEMD and tunable Q-factor wavelet transform," Mechanical Systems and Signal Processing, vol. 48, no. 1-2, pp. 103-119, 2014. 